Thorax (1950), 5, 207.

\title{
AN ANATOMICAL STUDY OF THE BRONCHIAL VASCULAR SYSTEM AND ITS VARIATIONS IN DISEASE
}

BY

PAUL MARCHAND, J. C. GILROY, AND V. H. WILSON

From the Thoracic Surgery Unit and Department of Medicine, Baragwanath Hospital, Johannesburg

During the course of an anatomical study of specimens of emphysematous lungs evidence was found to suggest that the bronchial circulation might play a more important part in producing the disease picture than has been appreciated hitherto. Consequently we undertook a detailed investigation of the bronchial arteries and veins in both normal and diseased lungs.

In some respects our findings differ from presently accepted views of the anatomy of these vessels.

\section{Material and Methods of Investigation}

The material used in our experiments is set out in Tables I and II.

The specimens have varied from very fresh lungs obtained at operation to necropsy material injected at least 24 hours after death and after rigor mortis had passed off. In all, a total of 63 lung specimens were injected.

The following injection techniques were employed:

The Vinylite Corrosion Technique (Puckett and Newman, 1940).-In this procedure coloured plastic was injected into the vessels under a positive pressure which varied from 2 to $5 \mathrm{lb}$. per sq. in. The organic material was digested off the cast by immersion in either concentrated hydrochloric acid or a $40 \%$ solution of caustic potash. After digestion a careful dissection was necessary to remove the finer radicles of the pulmonary vessels in order to visualize for study the bronchial circulation. The lungs, in the majority of instances, were inflated with air before the injection of the blood vessels ; in other cases vinylite was formed into the bronchi as an alternative to inflation with air. In preparing demonstration specimens, casts of the bronchi were made by pouring Wood's metal into the trachea before opening the chest.

When injecting complete lungs obtained at necropsy the pulmonary artery was filled through a cannula tied into the main stem; the pulmonary veins were filled through a cannula in the left auricular appendix, the aorta being ligated at its origin to prevent the escape of vinylite into the systemic vessels. The bronchial arteries were injected through a cannula tied into the divided descending aorta, the arch of the aorta being clamped at the origin of the left subclavian artery and the intercostal vessels being tied or clamped before or during the injection. The veins which enter the azygos and hemiazygos veins were injected through these veins in a retrograde manner. 
When dealing with specimens obtained at operation, injection was accomplished through the cut ends of the hilar vessels, and the bronchial artery was dissected out and cannulated directly. Foetal lungs were injected in the same way. The ductus arteriosus was ligated and the left auricle was clamped in such a way as to prevent the loss of injection mass through the foramen ovale and the atrio-ventricular valve. In these specimens the bronchial arteries were injected with a $7 \%$ solution of vinylite in acetone to ensure penetration of the finer vessels. In all our other injections in foetal and in adult lungs a $12 \frac{1}{2} \%$ solution was used. Both lungs were always injected in the necropsy specimens.

Perfusion of the arteries with saline before injecting the vinylite was not done because we found that this procedure caused waterlogging of the tissues with consequent occlusion of small vessels. Satisfactory injection casts of the pulmonary vessels can be obtained with vinylite after manual expression of clots from the larger vessels before injecting them. The specimens injected by this technique are tabulated (Table I).

TABLE 1

TABLE of Specimens InJeCted by the Vinylite-Corrosion Technique

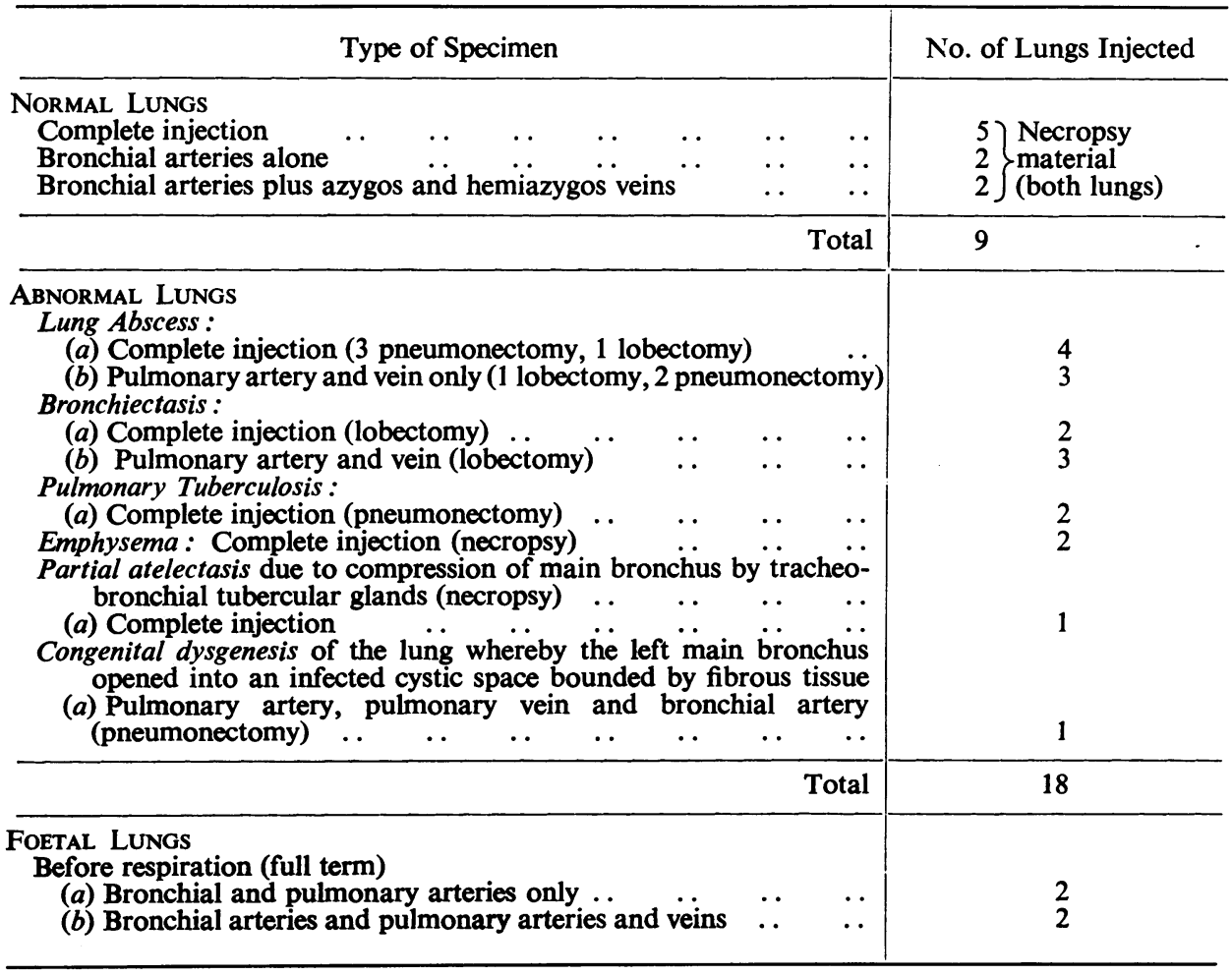

Injection of Radio-Opaque Media.-This method has been used to demonstrate the course and connexions of the bronchial arteries and of those veins which drain 
into the systemic circulation. The routes of injection have been the same as those employed for the vinylite injections.

We have used lipiodol (Lafay 40\%) $75 \%$ with $25 \%$ acetone but this solution, although it gives excellent radio-opacity, has the disadvantage of penetrating capillary vessels. Recently we have modified the formula of Hill (1929) who used a bismuth oxychloride suspension in gum acacia by substituting a saturated solution of sodium iodide for the water component of the original formula. This gives the solution a bright yellow colour which is easily recognizable and adds materially to its radio-opacity. The advantage of Hill's formula is that the solution will not penetrate the capillaries. The use of this method does not preclude subsequent injection with vinylite and both techniques have been used in the same specimen on three occasions (two normal lung specimens and one of emphysema). We felt that the penetration of the bronchial arterial injection with vinylite might not be complete if it were preceded by an injection of bismuth oxychloride. We have therefore made it our usual practice to use one or other method alone.

After completion of the injection, stereoscopic radiographs of these specimens were taken at four feet. These lungs were finally dissected, a special effort being made to follow the bronchial artery and its branches as far into the substance of the lung as possible.

The specimens treated by this method are tabulated in Table II.

TABLE II

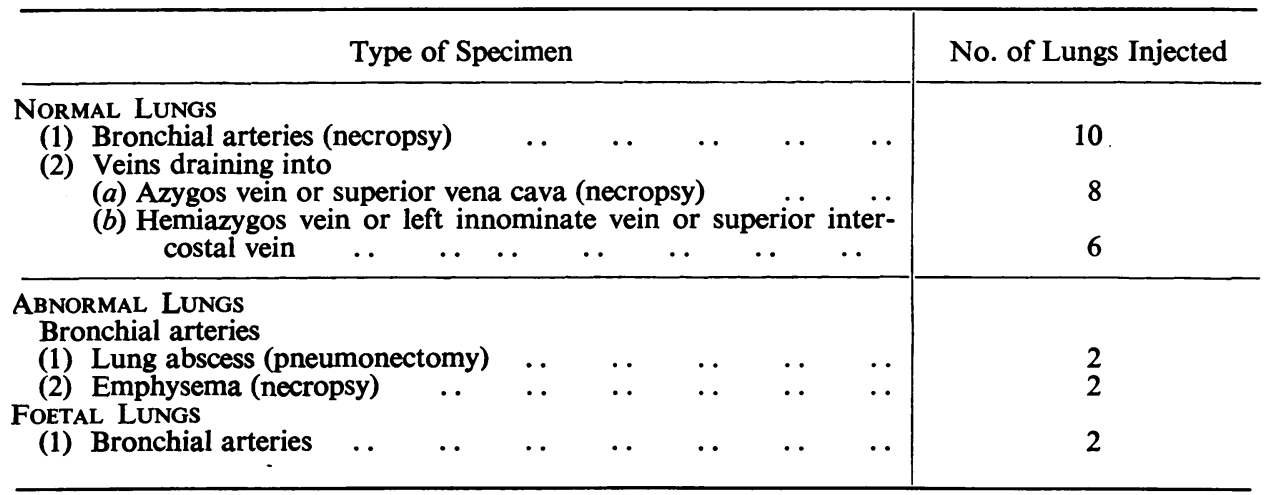

Injections with Gelatine.-Two specimens of emphysema and two normal lungs have been injected through the main pulmonary artery with a carmine gelatine preparation after the formula of Moore (1929) to which we added $20 \%$ yellow oxide of lead in order to obtain radio-opacity. Serial sections of the bronchi and bronchioles were cut and examined.

\section{RESULTS OF INJECTION EXPERIMENTS IN SPECIMENS OF NORMAL LUNGS}

The bronchial arteries show some inconstancy in their origin, distribution, and course, though this has been less than we expected. The commonest site of origin is from the antero-lateral aspect of the aorta about $\frac{1}{2}$ in. to 1 in. distal to the origin 
of the left subclavian artery. Usually the arteries to the right and left lungs arise separately although they may have a common origin from the aorta. Either artery may arise from an intercostal artery, and Miller (1947) states that the bronchial artery may originate from the internal mammary artery.

There are usually two main arteries, one to each lung. In addition, one or more smaller arteries arise directly from the aorta about another half inch distal to the main bronchial arteries, but they may arise from any part of the aorta above the diaphragm (Fig. 1).

Where the lung is supplied by more than one bronchial artery the upper and

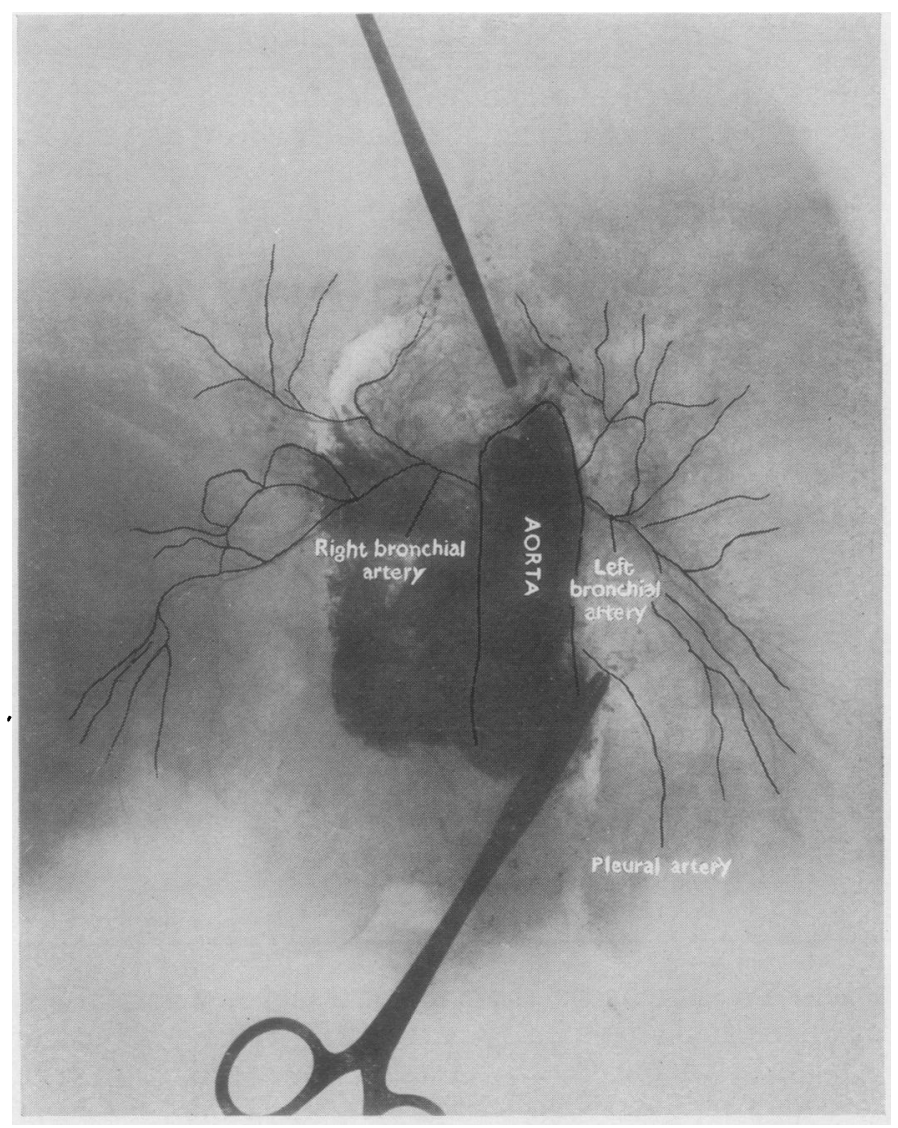

FIG. 1.-Injection through the aorta of the bronchial arteries of a normal lung with radio-opaque bismuth suspension. This is one of the two lungs that showed no pulmonary arterial filling. Both lungs are supplied by a single main bronchial artery, the point where the right bronchial artery divides being obscured by the smudged area where the medium has escaped into interstitial tissue. Note how the arteries branch and re-anastomose after the main artery has entered the hilum and given off branches which run with the main segmental bronchi. normally placed vessel supplies the greater portion of the lung; the additional artery seldom supplies more than the posterior and medial aspects of the lower $10 \mathrm{be}$, although in one of our specimens it supplied most of the right lower lobe. The additional bronchial artery traverses the pulmonary ligament, and the lower its origin from the aorta the more limited seems to be its distribution. This artery may be the source of annoying, and sometimes of troublesome bleeding, when the pulmonary ligament is divided during pneumonectomy or lower lobectomy.

Several small arteries which arise either directly from the aorta or from the extra-pulmonary course of the 


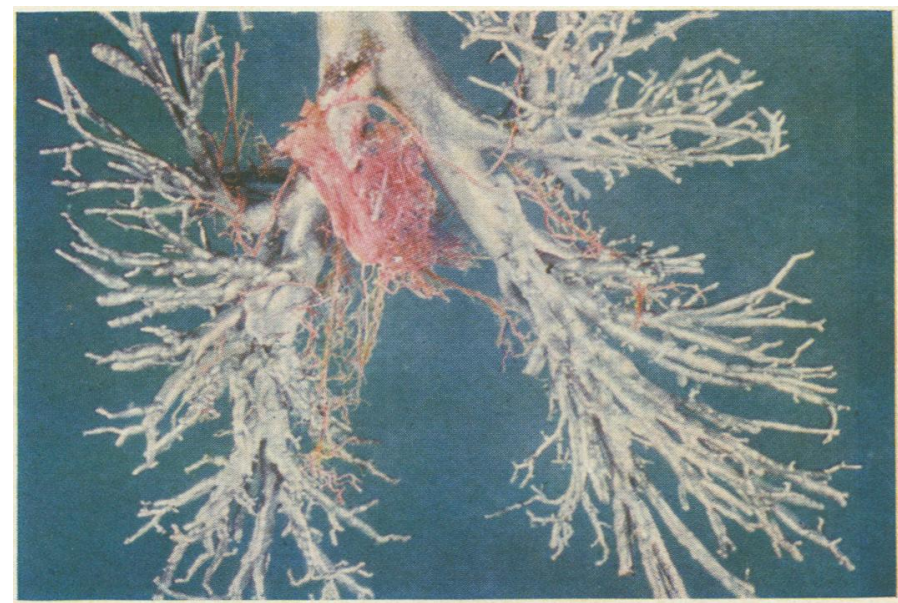

FiG. 2.-Wood's metal cast of bronchial tree. Red vinylite was injected into the aorta, and shows the manner of origin of the bronchial arteries as they arise from the aorta. In this specimen both lower lobes are partly supplied by accessory bronchial arteries arising from the aorta a few centimetres distal to the main bronchial arteries. This specimen is photographed from behind and shows the aorta crossing the left main bronchus.

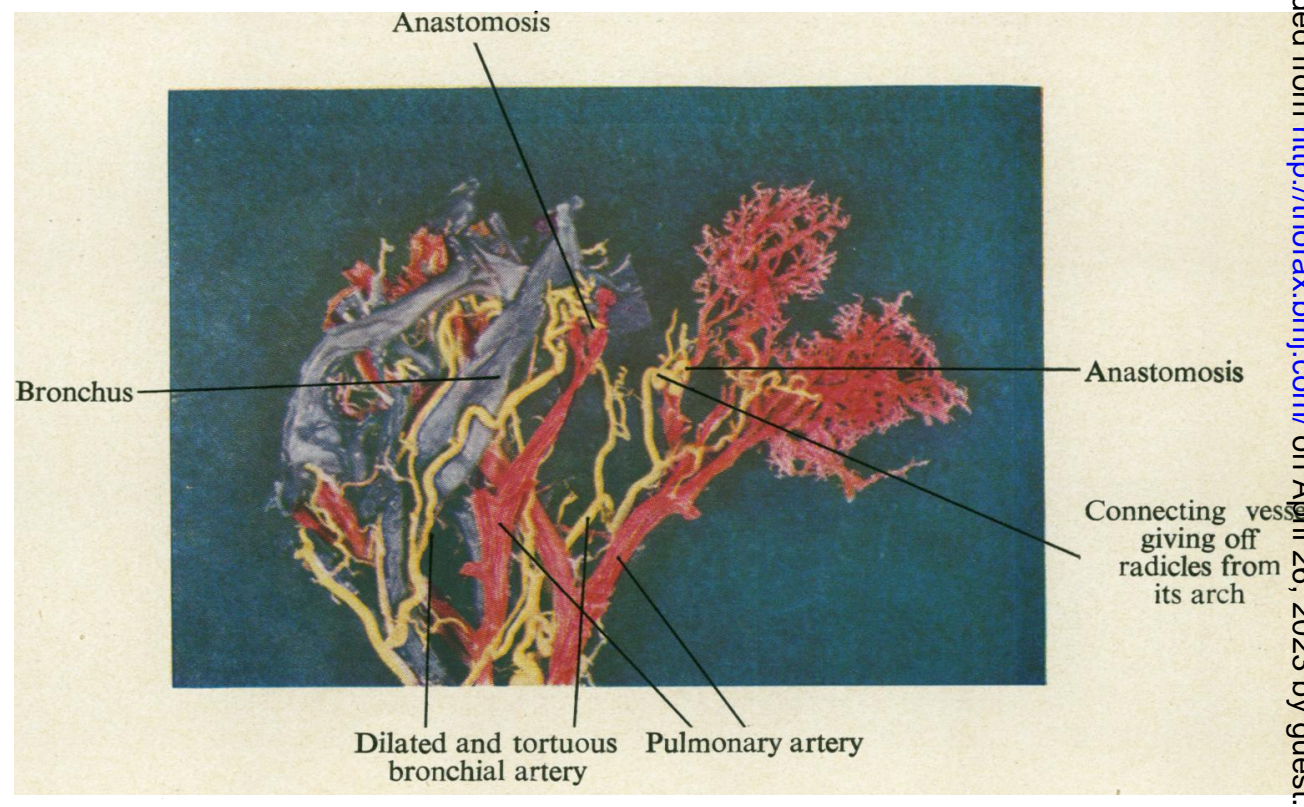

Fig. 4.-Vinylite cast of the subapical segment of the right upper lobe of an emphysematous lung. The bronchi show up blue, the bronchial arteries yellow, and the pulmonary artery red. This specimen has also been flattened by placing in warm water. There are several anastomoses to be seen demonstrating the dilatation and tortuosity of the parent bronchial arteries, the radicles arising from the arch of the connecting vessel, and the manner in which the connecting vessel arises from the parent pulmonary artery. The bronchi have been removed from the right-hand portion of the specimen.

[Facing page 210 
bronchial artery, pass to the mediastinal aspect of the lung and then spread out subpleurally. Neither the bronchial nor the intercostal arteries leave the aorta at right angles to its axis, but they pass upwards initially forming an acute angle with the aorta (Fig. 2). During systole the effect of the elongation and dilatation of the aorta is to accentuate this angulation.

The main bronchial arteries usually arise from the aorta just after it has crossed behind the left main bronchus, the artery to the right lung passing upwards to cross the mid-line at a point level with or just below the tracheal bifurcation. Both arteries enter the lung closely applied to the membranous portion of the main stem bronchus. The main bronchial artery continues along the posterior aspect of the main stem bronchus. Branches of this vessel are then given off to supply each main segmental bronchus, the anteriorly placed ones being reached by winding round the lateral aspect of the main bronchus. These branches are intimate relations of the outer wall of the bronchus.

The mediastinal pleura is prolonged into the lung with the bronchi, and by careful dissection the bronchial arteries can be followed well into the substance of the lung without coming in contact with the lung parenchyma; they lie between the invaginated pleura and the fibrous layer covering the bronchi. The invaginated pleura fuses with the outer bronchial coat at the origin of the segmental bronchi. If the bronchial arteries are followed further than this point the interstitial tissue of the lung itself must be exposed. The bronchial arteries are now truly intrapulmonary and from here onwards branch repeatedly and by re-anastomosis form an arterial mesh around the bronchi (Fig. 1). Small branches constantly pierce the bronchial wall to form an intramural network as described by Miller ((1947). In normal lung specimens the bronchial arteries cannot be traced by our methods further along the surface of a bronchus than the third dividing point of a segmental bronchus.

The arteries which arise from the aorta distal to the origin of the left subclavian artery include bronchial, pleural, and mediastinal arteries, and these supply the tracheo-bronchial tree from the thoracic inlet as far as the respiratory bronchiole. They also supply the tracheo-bronchial and interbronchial lymph glands and vasa vasorum to the pulmonary vessels; these vasa vasorum never communicate with the lumen of the pulmonary artery. Branches from these vessels also pass along the interlobular septa to supply the visceral pleura of the interlobar fissures. We have been unable to demonstrate a bronchial arterial supply to the greater part of the costal portion of the visceral pleura and this agrees with the findings of von Hayek (1940) and Verloop (1948). This area of the pleura is supplied by the pulmonary artery.

The existence of macroscopic anastomoses between bronchial and pulmonary arteries has been denied by Miller (1947), but Braus (1931) and Liebow, Hates, and Lindskog (1949) have demonstrated such anastomoses in normal and diseased lungs. We have demonstrated them in three of the four normal lung specimens in which both the bronchial and pulmonary arteries were injected with vinylite, one of these specimens being the lungs of a child aged two, who had died from meningitis. We have also demonstrated our radio-opaque medium (which will not pass through capillaries) in the pulmonary arteries of eight of ten normal lung specimens in which the bronchial arteries alone were injected. 
These anastomoses have a constant anatomical design. As the bronchial artery approaches the pulmonary artery with which it is to anastomose it becomes widened and tortuous and gives off a branch which arches over to enter the pulmonary artery

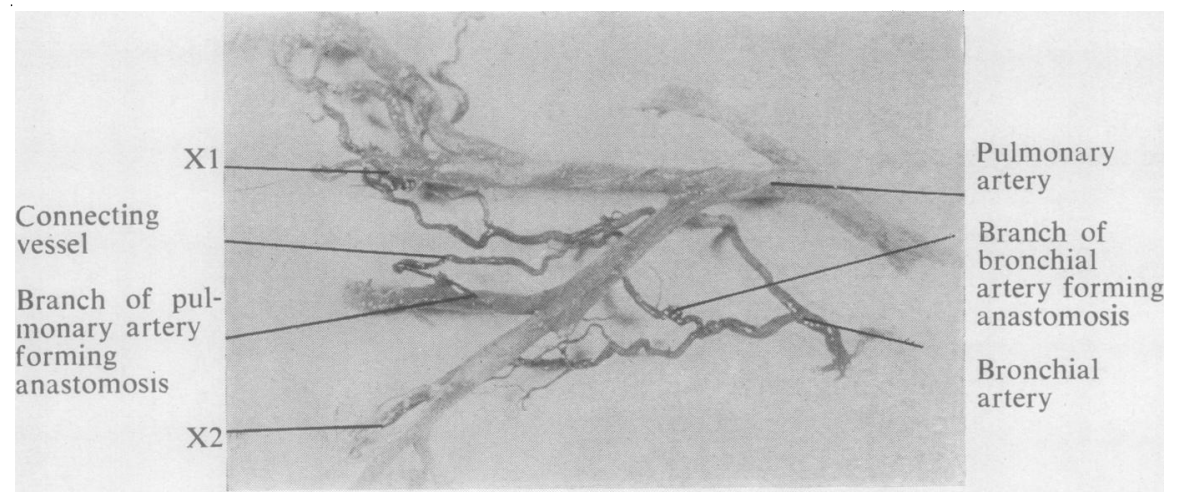
Fig. 3.-Isolated seg- ment of a vinylite cast of pulmonary artery and bronchial artery from a lobe which was from a lobe which was the site of a lung abscess. The specimen has been placed in warm water and flat- tened so as to photo- graph more readily. The bronchial artery, giving rise to the con- giving rise to the con- necting vessel, showed much greater cork- screw tortuosity before this procedure. This specimen shows the manner in which the connecting vessel arises from both arises from both bronchial and pul- monary arteries. $\mathrm{O}$ ther anastomoses marked $X 1$ and $X 2$ are present but do not show up distinctly.

against the direction of the latter's blood flow (Fig. 3). The branch which actually effects the anastomosis has been termed the connecting vessel. In all cases this connecting vessel could as well be considered a branch of the pulmonary artery as of the bronchial artery (Fig. 3). From the arch of the connecting vessel small radicles are given off which pass along the bronchial wall (Fig. 4). Rivkind (1949) also commented upon these branches of the anastomotic vessel.

The vinylite injection technique will only demonstrate those anastomoses which occur on the surface of the bronchi because a $12 \frac{1}{2} \%$ solution of vinylite will not enter vessels with a diameter of less than 200 microns. The anastomoses were chiefly in the region of the bronchi which still possess a cartilaginous wall. The method is a crude one only capable of demonstrating anastomoses with the widest calibre and probably but a fraction of those actually present. For this reason we have made no attempt to measure the size of the vessels involved in the anastomoses, or to count the numbers present in any particular segment of lung.

\section{The Bronchial Artery in Foetal Lung}

The origin and distribution of the bronchial artery in the full term foetal lung is the same as that already described. In the atelectatic lung of the stillborn infant, the bronchial artery is a well developed structure with a calibre not much smaller than that of the adult artery. When compared with the main pulmonary arteries of the foetus its relative size is proportionally much greater than that seen in the adult.

We have been unable to demonstrate macroscopic arterial anastomoses between the bronchial and pulmonary circulations in the lungs of a stillborn infant using the vinylite injection technique, but in those specimens in which the bronchial arteries were injected with radio-opaque medium the dye could be demonstrated in the pulmonary artery. We estimate that the bismuth oxychloride medium will not enter 


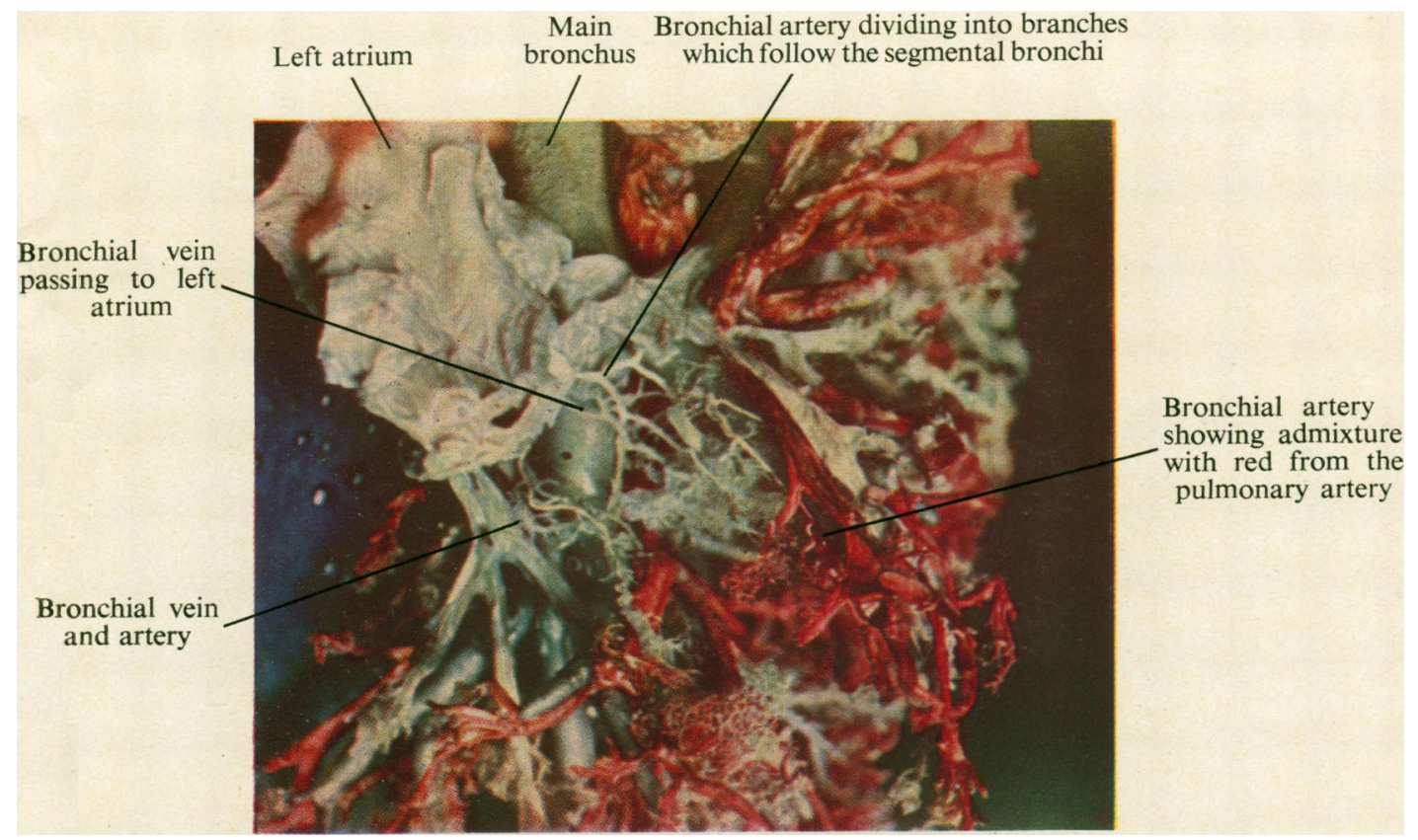

Fig. 5.-Complete cast of a normal left lung of an infant. The bronchi are filled with Wood's metal. The pulmonary artery has been injected red, the bronchial artery yellow, and the left atrium, pulmonary veins, and bronchial veins blue. (The atrium and pulmonary veins appear whitish in parts because of the bleaching (The atrium and pulmonary veins appear whitish in parts because of the bleaching
action of the hot photographic lamps.) This specimen shows the manner of division of the bronchial arteries and a bronchial artery giving rise to a connecting vessel. (The actual anastomosis is hidden, but the admixture of red and yellow vinylite is clearly shown.) The termination of the bronchial veins in the atrium is obscured by the atrium itself.

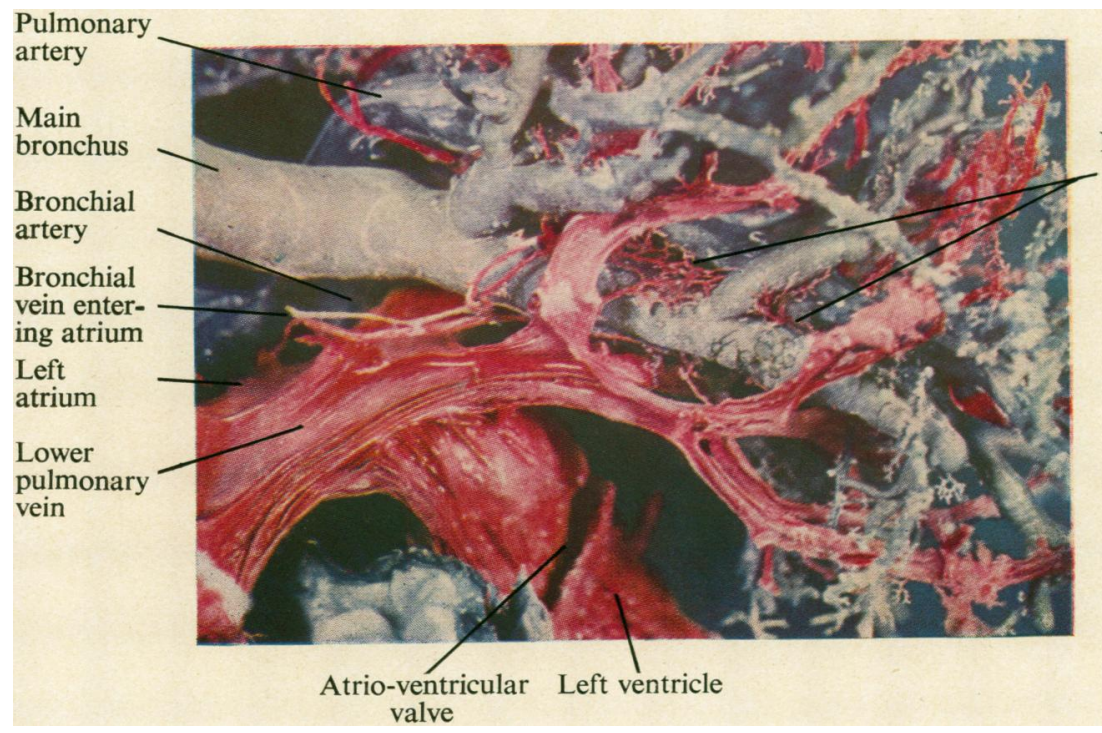

Network bronchia venules

Fig. 6.-Vinylite and Wood's metal cast of section of the lung of a male aged 50 in whom the heart and lungs were normal. The pulmonary artery was injected with blue vinylite. The left atrium, and through this the left auricle, pulmonary veins, and bronchial veins were injected with red vinylite. The bronchial artery was injected yellow but has been removed, except for a small segment, so as to demonstrate the bronchial vein around the segmental bronchi and extending over the lower lobe bronchus. The main bronchial vein can be seen entering over the lower lobe bronchus. The main bronchial vein can be seen entering
the left atrium and its size can be compared with that of the adjacent bronchial artery. 
vessels less than 20 microns in diameter yet the distinctive yellow colour of the suspension, which appeared in the main pulmonary artery during the injection of the bronchial arteries was unmistakable, and indicates the presence of pre-capillary anastomoses between bronchial and pulmonary arteries.

\section{The Bronchial Veins}

Miller (1947) stated that true bronchial veins are found only at the hilum of the lung and that they arise "from the first or the first two dividing points of the bronchial tree," and that they may also receive branches from that part of the pleura in close proximity to the hilum. He describes these veins as draining into the azygos, hemiazygos, or one of the intercostal veins. Within the lung he mentions venous plexuses in the walls of the bronchi and bronchioles which unite at the bifurcation of a bronchus or bronchiole to form a radicle which empties into the pulmonary vein.

We have found that two distinct groups of veins exist, namely, the deep or true bronchial veins which are intrapulmonary vessels related to the bronchi and which drain into the pulmonary veins or into the left auricle direct, and a second group of veins which drain the subpleural and hilar structures into the azygos and hemiazygos veins as described by Miller (1947). Miller makes no mention of the extensive communication between this second group of veins and the pulmonary veins.

On the basis of our findings we would suggest the following nomenclature.

The Deep or True Bronchial Veins.-Whenever vinylite was injected through the left auricular appendix in order to fill the pulmonary veins we were able to demonstrate these bronchial veins. They first appear as macroscopic structures in the region of the terminal bronchiole. At this level they appear as a very fine lacy network surrounding the bronchiole, and they become more obvious by the accession of further radicles from the intrabronchial venous plexuses. Eventually they are seen as several vessels running longitudinally along the outer wall of the larger bronchi (Fig. 5). Near the hilum the several vessels unite to form a single trunk which is slightly larger in diameter than the bronchial artery, and this vein terminates either in the atrium direct (Fig. 6) or into a main pulmonary vein clos? to the atrium. If the injection to demonstrate these veins is not made via the left atrium, their mode of termination may easily be missed.

We have found one main deep bronchial vein in each lung. On the left side it forms an anterior relation to the main stem bronchus just below the origin of the left upper lobe bronchus. If it enters the atrium it does so anywhere between the two pulmonary veins but usually close to the lower one.

The mode of termination of the bronchial vein on the right side is essentially the same, but because of the high situation of the right upper lobe bronchus, it crosses the lower part of the right main bronchus in relation to the middle lobe bronchus.

Throughout their intrapulmonary course these bronchial veins communicate freely with the pulmonary veins.

The Pleuro-Hilar Veins.-There is a rich subpleural network of veins, most of which pass towards the mediastinum along the anterior and posterior surfaces of 
the lung, and drain into one or more veins which run beneath the pleura at the junction of lung and mediastinum. These latter veins are present on both the anterior and posterior surfaces of the hilum. A limited area of the visceral pleura however drains into the pulmonary vessels. Apart from the pleural vessels the veins at the junction of lung and mediastinum receive tributaries from the extrapulmonary bronchi, the hilar lymph glands and the vasa vasorum of the pulmonary ressels at the hilum. "Pleuro-hilar veins" seems an appropriate name as it describes the regions whose venous drainage passes into these veins. The veins on the right side usually empty into the azygos vein just before that vessel enters the superior vena cava. In two specimens the termination has actually been into the latter vessel.

The veins on the left enter the hemiazygos, the superior intercostal or the left innominate vein. As the veins on the left side have this somewhat inconstant termination, they are often difficult to demonstrate.

In every lung injected through these pleuro-hilar veins, we were able to show free filling of the pulmonary veins. Communications between the pleuro-hilar and pulmonary veins occur at the hilum and in the subpleural areas. The communication at the hilum is via veins in the region of the hilar bronchi. These are essentially extrapulmonary in character as they lie in the potential space between invaginated pleura and the dense outer coat of the bronchi. These are the bronchial veins described by Miller (1947). They are bronchial veins in the sense that they drain the large bronchi at the hilum, but their only connexion with the intrapulmonary bronchial venous system is an indirect one by way of communicating vessels to the pulmonary veins, and probably by a limited connexion with the intramural venous plexuses.

The venous arrangement is therefore one whereby the intrapulmonary bronchi and bronchioles drain via the deep or true bronchial veins and empty into the pulmonary venous system (we include the left atrium in this system), whereas the subpleural areas and the hilar structures drain into systemic veins by a separate system of vessels which communicate freely. with the pulmonary veins.

As previously stated, the arterial supply to the hilar and subpleural structures is usually quite separate from the bronchial artery, and therefore we prefer to term both the arteries and the veins "pleuro-hilar vessels." If an accurate description of function is to be achieved, the term "bronchial " should only be applied to those vessels which supply and drain the intrapulmonary portions of the bronchial tree.

\section{THE BRONCHIAL VASCULAR SYSTEM IN DISEASED LUNGS LUNG ABSCESS AND BRONCHIECTASIS}

Sections cut through the wall of an acute lung abscess or from the surrounding area of pneumonitis show that the radicles of the pulmonary artery are obliterated by infected thrombi. In chronic lung abscess organized thrombi with evidence of recanalization can be shown. In vinylite casts the pulmonary artery and veins demarcate the abscess area distinctly (Fig. 7). It will be noted that the vascular pattern in the abscess areas has been altered. Very little pulmonary artery remains 


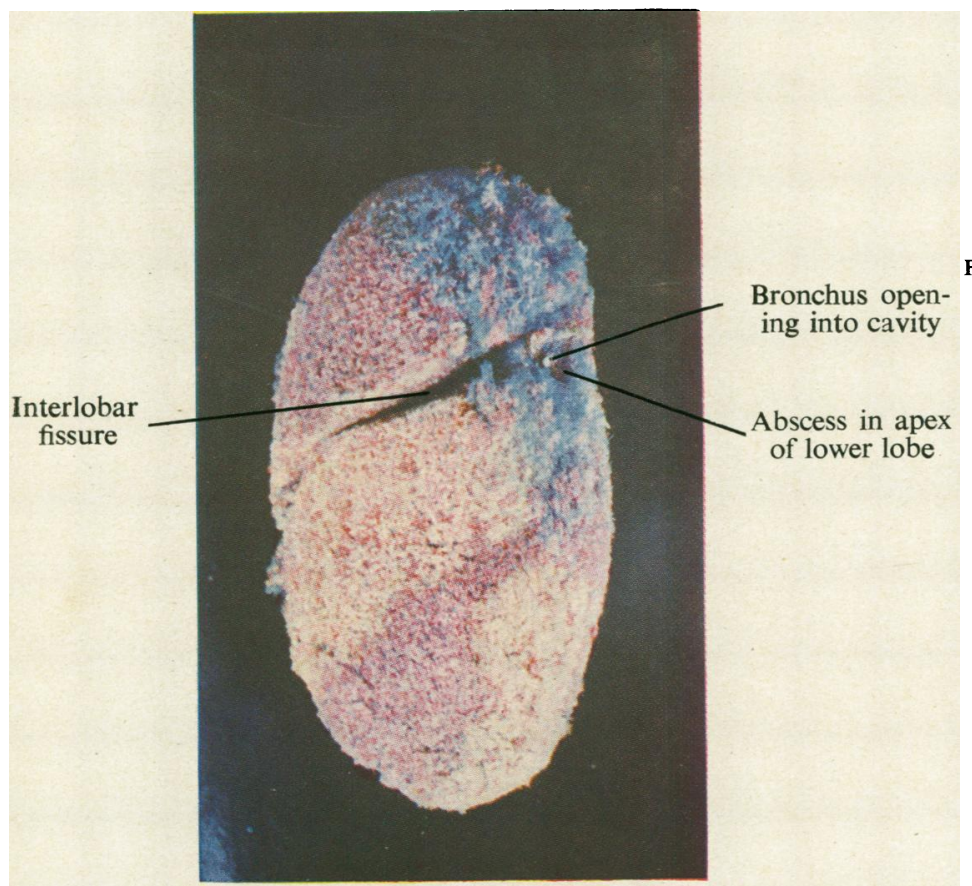

FIG. 7.-Vinylite cast of the left lung removed at operation for two chronic lung abscesses, one in the subapical area of the upper lobe and the other in the apex lobe and the other in the apex
of the lower lobe. The bronchi of the lower lobe. The bronchi the pulmonary arteries red, and the pulmonary veins blue. The two abscess areas, separated by the interlobar fissure, are clearly the interlobar fissure, are clearly demarcated by the blue of the pulmonary vein. Very little pulmonary artery is to be seen in the abscess areas. The cavity in the apex of the lower lobe can be seen. The yellow mass of vinylite which occupied it has been removed. The area of pulmonary arterial destruction extends for some distance around the actual cavity. The bronchial the actual cavity. The bron

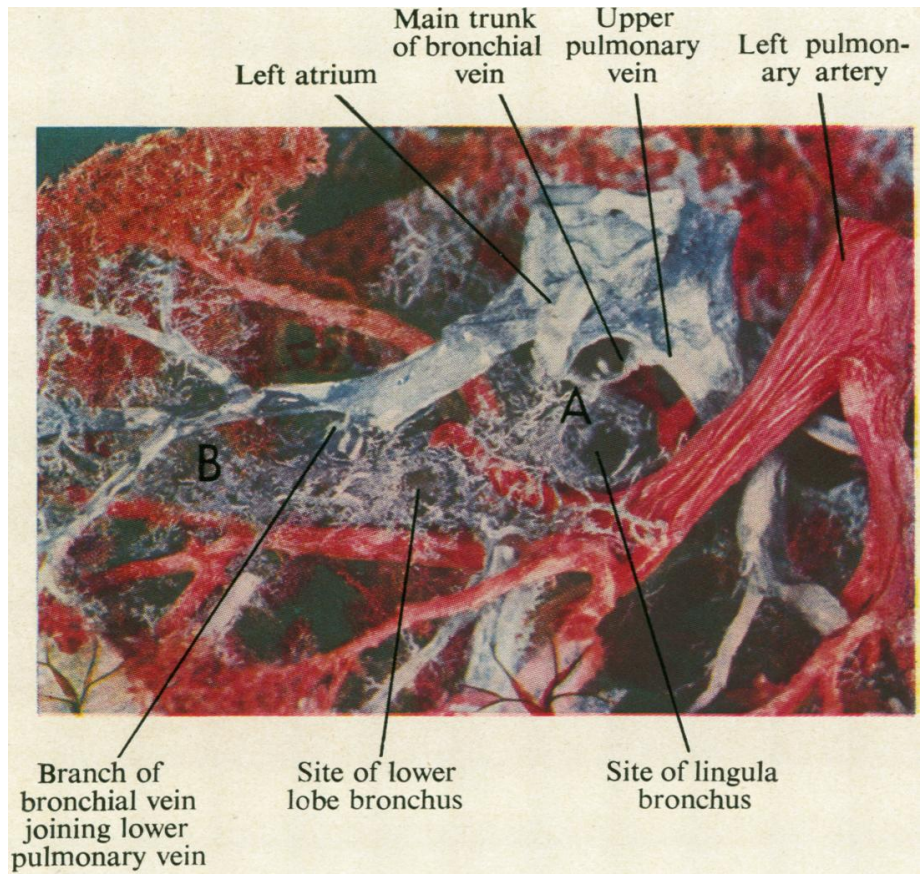

FIG. 8. -Vinylite cast of the left lung from a case of emphysema associated with cor pulmonale. (Pulmonary artery red, pulmonary veins, bronchial veins, and left atrium blue.) The bronchi were not injected, but their course is outlined by the atrium blue.) marked A and B. Not adequately visualized is the extension of the bronchial veins along the course of the upper lobe bronchi and along the branches of the main bronchus.

[Facing page 214 
and consequently the abscess areas are demarcated by the blue dye in the pulmonary veins. Injection of the bronchial artery demonstrates that the arterial supply to the abscess area is provided by this vessel.

Macroscopic anastomoses between the bronchial and pulmonary arteries are plentifully demonstrated; these are seen not only immediately adjacent to the abscess, but are widespread throughout the affected lobe. They are also increased in the remaining, apparently normal lobes of a diseased lung. Anatomically the anastomoses are identical with those found in normal lungs, being situated mainly in the region of the smaller bronchi.

In cases of early bronchiectasis in which the infection is limited to the wall of the bronchus, the number of anastomoses found is not noticeably increased, but once infection spreads to the parenchyma of the lung and consequent fibrosis has occurred, the anastomoses become conspicuous and widespread throughout the affected lobes. Wood and Miller (1937-8) and Liebow et al. (1949) have also described arterial anastomoses in cases of lung abscess and bronchiectasis.

Two specimens of pulmonary tuberculosis, affecting both lungs and in which fibrosis was widespread, also displayed a similar increase in the number of arterial anastomoses.

\section{Generalized Pulmonary Disease Associated With Cor Pulmonale}

Three autopsy specimens of emphysematous lungs, one of which was associated with long-standing atelectasis of the left lung, the right lung being markedly emphysematous, were injected with vinylite. In a further two cases of emphysema the pulmonary arteries were injected with gelatine. The lung pathology has been confirmed microscopically in all cases. All the patients from whom these specimens were obtained exhibited the cardiac signs of cor pulmonale during their illness.

The Bronchial Artery.-The vinylite casts show broncho-pulmonary arterial anastomoses to be widespread throughout both lungs, including the partially atelectatic lung. These anastomoses were structurally identical with those found in normal lungs. A striking feature of these anastomoses was the calibre of the connecting vessel which often approximated that of the pulmonary radicle which it entered. This is not peculiar to the lungs of cor pulmonale because we have seen it in specimens of lung abscess and even occasionally in specimens of normal lungs (Fig. 4).

The two lungs injected with gelatine must also have had widespread arterial anastomoses because the gelatine which was injected into the pulmonary artery appeared in considerable amounts in the aorta by way of the bronchial arteries.

The Bronchial Veins.-Whereas normally the true bronchial veins are delicate vessels, our vinylite casts of the vessels in generalized lung disease showed a very conspicuous venous network outlining the course of the bronchi. The longitudinal venous trunks united to form main vessels two or three times as large as normal ones. The connections with the pulmonary veins were abnormally dilated and easily recognizable (Fig. 8). Serial section of the smaller bronchi in the gelatine-injected lungs revealed dilatation of the intra-bronchial venous plexuses within the walls of the bronchi. 


\section{DISCUSSION}

The question as to whether or not the bronchial artery anastomoses with the pulmonary artery, has been the subject of much controversy. Miller (1934), who worked with dogs, stated that pre-capillary anastomoses did not exist, and he expressed the opinion that vasa vasorum to the pulmonary vessels were being mistaken for true anastomoses. We cannot agree with this, and our findings together with those of Braus (1934), von Hayek (1940), and Verloop (1948) prove that broncho-pulmonary arterial anastomoses exist in normal human lungs. The vinylite technique will only demonstrate the anastomoses between the comparatively large arteries of the bronchial and pulmonary systems. Verloop (1948) made a detailed histological study of the arteries forming these anastomoses in normal human lungs and described pre-capillary anastomoses along the surface, and within the walls, of all the smaller bronchi. In addition there are superficial anastomoses between bronchial and pulmonary radicles under the visceral pleura, though these are far less numerous than the anastomoses related to the bronchi. He concluded that the total number of broncho-pulmonary anastomoses must be very great.

Verloop (1948) also found anastomoses in foetal lungs but remarks on the fact that they were few in number. From our experiments with radio-opaque bismuth oxychloride injection we are in agreement that anastomoses between bronchial and pulmonary arteries are already present in foetal lungs. In addition to pre-capillary anastomoses there is probably an extensive capillary anastomosis between pulmonary and bronchial arteries which subsequently become arterialized to form the very extensive anastomatic system found in the adult.

The increase in the number of macroscopic arterial anastomoses found in diseased lungs, as compared with normal, must be due to dilatation of existing anastomoses. They cannot be a direct result of revascularization following infection or necrosis, because in specimens of lung abscess they occur remote from the affected lung segments. They are also increased in emphysematous lungs, a generalized disease in which local infection and necrosis are absent. Apart from the numerical increase, the macroscopic anastomoses found in diseased lungs are structurally identical with those in the normal. The function of these anastomoses is still under investigation, but there is little doubt that the dilatation is due to the passage of an increased volume of blood through the anastomoses which therefore appear to be concerned with the redistribution of blood flow within the lungs.

With the exception of von Hayek (1940) all recent workers agree that blood must flow from bronchial to pulmonary artery when the anastomoses become functionally active. This conclusion is based upon supposed pressure differences between the two systems. Fig. 9 represents diagrammatically our views upon the possible effects the broncho-pulmonary anastomoses have upon the distribution of blood flow within the lungs.

Normally there is probably little interchange of blood between the two systems due to constriction of the wall of the muscular connecting vessel (Fig. 9-(D).

Bloomer, Harrison, Lindskog, and Liebon (1949) showed that after ligation of the pulmonary artery in dogs, oxygenated blood circulated through the pulmonary arteries distal to the point of ligature. If the bronchial artery and the connecting 

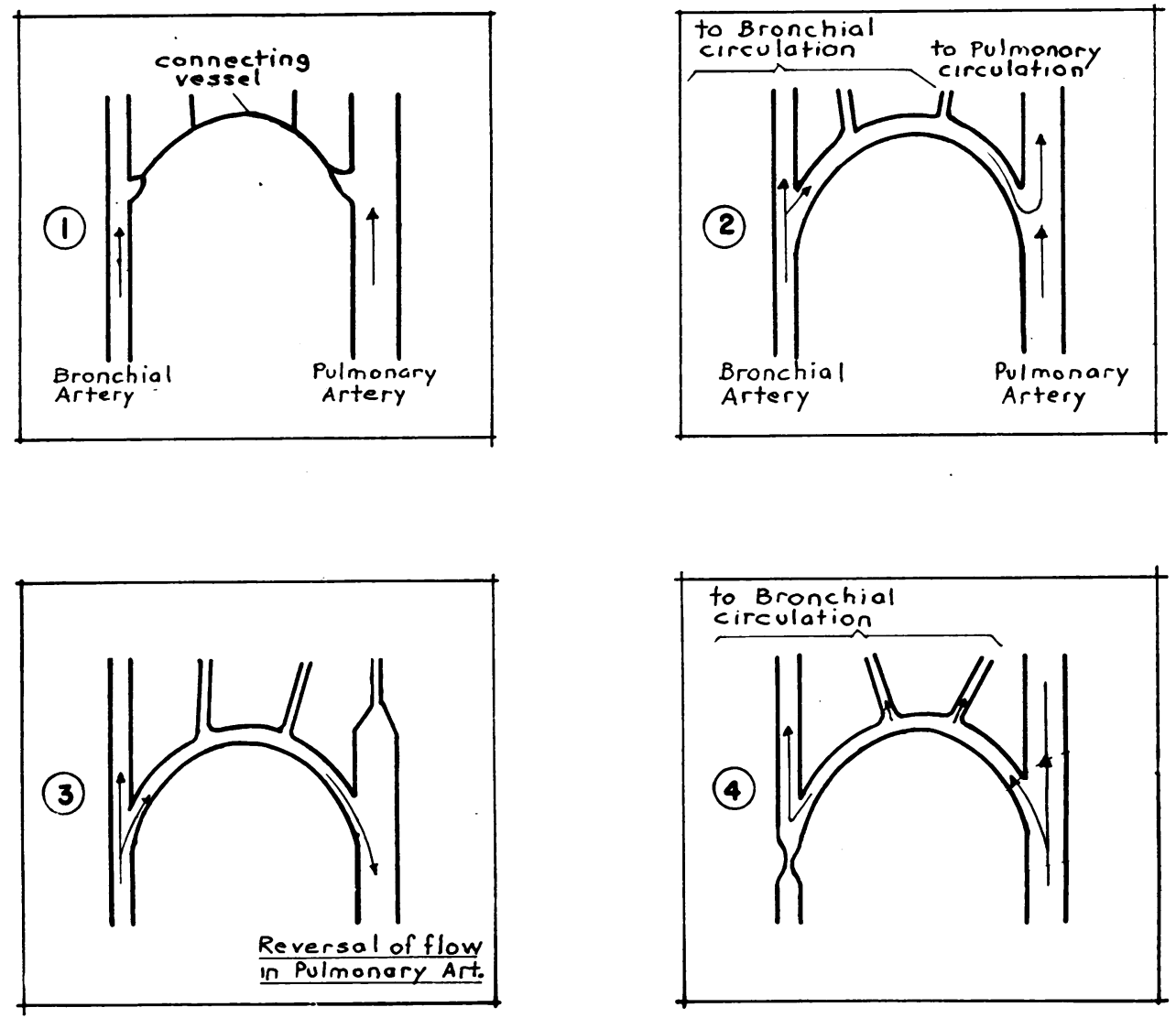

FIG. 9.-Diagrammatic representation of possible interchange of blood between pulmonary and bronchial circulations.

vessel are patent and there is obstruction to the pulmonary artery, such as was produced in Bloomer's experiment, the pressure within the pulmonary artery distal to the obstruction will drop and bronchial blood will be directed into the pulmonary circulation. Virchow (1851) demonstrated that ligation of the pulmonary artery did not result in gangrene of the lung and the arterial anastomoses functioning in this direction (Fig. 9-(2) may be an important factor in preventing necrosis following a pulmonary embolus.

The main source of oxygenated blood to the foetal lung is probably via the bronchial arteries reaching the pulmonary alveolar capillaries through the arterial anastomoses.

East and Barnard (1938) published a description of four cases of Fallot's tetralogy which at necropsy showed complete stenosis of the pulmonary artery, yet the affected individuals survived until the ages of $33,30,20$, and six years respectively. The bronchial arteries were the sole source of pulmonary blood and in two were grossly dilated. Blood could only have reached the alveolar capillaries through 
these anastomoses passing from bronchial to pulmonary artery. We have been unable to study the bronchial arteries in a case of Fallot's tetralogy at necropsy but they are probably frequently dilated. The exposure of the pulmonary artery in the course of Blalock's operation for the relief of Fallot's tetralogy is often difficult, because of the overlying plexus of dilated vessels. These vessels have been mentioned by Keith (1909) and by Fatti and Gilroy (1949) and are probably derived from the pleuro-hilar arteries which arise direct from the aorta or from the extrapulmonary portion of the bronchial artery. Verloop (1948) has demonstrated that arterial anastomoses between the pleural and pulmonary arteries occur in the subpleural areas, and it is along this pathway that systemic blood will reach the pulmonary arteries. This is precisely what Blalock's operation accomplishes on a larger scale.

Fig. 9-(3) illustrates the direction of blood flow in conditions associated with obstruction of the small pulmonary arterial radicles such as occur in atelectasis. This has been demonstrated by blood oxygen estimations on samples of blood taken at operation and the results will be reported fully in a further paper. If the atelectasis is lobular or lobar, blood is diverted into the pulmonary arterial branches of the remaining segments of lung. With total collapse of a lung the oxygenated arterial blood derived from the bronchial arteries, may actually be diverted to the other lung.

In all the above-mentioned cases blood has been diverted from bronchial to pulmonary artery and this has been accompanied by an increased delivery of blood from the aorta to the bronchial arteries. Under such circumstances one would expect to find the extrapulmonary bronchial artery to be dilated. We have demonstrated numerous and widespread broncho-pulmonary arterial anastomoses in lungs with tuberculosis, lung abscess, and emphysema, especially when associated with cor pulmonale. This has been taken as a probable indication that in these conditions an appreciable volume of blood crosses from one system of arteries to the other. In none of these latter specimens however was the extrapulmonary portion of the bronchial artery dilated. We have considered the possibility of the passage of blood from pulmonary to bronchial artery, a possibility which only von Hayek (1940) has entertained. All other workers consider that hydrostatic pressure differences between the two systems would preclude pulmonary arterial blood from entering the bronchial artery. We are not convinced that such pressure differences do, in fact, exist. We have been unable to cannulate the bronchial artery at operation, but on occasions when it has been cut during the course of lobectomy, we have noticed that it does not spurt with the vigour of an artery such as the digital. Also the anastomoses occur in the substance of the lung arising from small branches of a bronchial artery which has divided and re-anastomosed repeatedly. It is possible that at the site of the anastomoses the pressures in the pulmonary and bronchial arteries are approximately equal. Apart from these considerations, the construction of the bronchial artery in the anastomatic area is such as to make pressure differences of little consequence in regulating the direction of blood flow. Von Hayek (1940), Mauer (1941), van der Zwaag (1940), and Verloop (1948) have described thick-walled muscular bronchial arteries within the lung, different from the extrapulmonary bronchial artery which histologically is typically systemic in 
structure. Verloop (1948) made a painstaking study of the histology of the connecting artery and its parent vessels, and he found that the segment of bronchial artery which gives rise to the anastomatic vessel always has an hypertrophied muscular coat. This hypertrophy mainly involves the longitudinal muscle layer within the intima, but the circular muscle in the media is also frequently increased. Von Hayek (1940) also comments upon the very muscular wall of the bronchial arteries which give off connecting branches to form anastomoses with the pulmonary arteries. Both workers have found that these bronchial arteries are capable of active occlusion of their lumina. The connecting vessel has a thinner wall than its parent bronchial vessel, but does possess substantial muscular fibres which run longitudinally. The pulmonary artery has only a few circular muscle fibres in its media and these are separated by thick elastic laminae. As Brenner (1935) remarks, the smaller pulmonary arteries seem best adapted to follow passively changes in the pulmonary blood pressure. They can have very little active control by virtue of their vaso-motor powers. The bronchial arteries and connecting vessels on the other hand, seem admirably designed to respond to vaso-motor control. If therefore, the bronchial artery proximal to the anastomoses is occluded by muscular constriction of its wall, blood could be diverted from the pulmonary to the bronchial artery (Fig. 9-(4)). Under these circumstances possible differences in hydrostatic pressure would not be operative. The function of the anastomosis in such an event would appear to be in the nature of an attempt to prevent the development, or minimize the effects, of pulmonary hypertension, and a consequence would be to divert unoxygenated pulmonary arterial blood into the bronchial circulation. We believe that blood does flow in this direction across the anastomoses between pulmonary and bronchial arteries in conditions of raised pulmonary arterial pressure. In localized disease the anastomoses are sufficient to counteract the effect of the disease process on the pulmonary arterial pressure and so prevent strain upon the right ventricle. In generalized disease, even though the anastomoses are widespread they may fail eventually in the function of obviating pulmonary hypertension. Wood and Miller (1937) remark that broncho-pulmonary anastomoses are most marked in heart disease with right-sided hypertrophy. We have found in vinylite casts that the density of the anastomoses in a particular segment of lung from a case of cor pulmonale is no greater than that in a segment affected with lung abscess. In the former, however, these macroscopic arterial anastomoses are widespread throughout both lungs.

Whatever the direction of the blood flow, the arterial anastomoses appear to be functionally significant in many varied forms of diseased lungs. The components of the anastomosis seem to have dilated in response to the continued increase in the blood flow traversing them from one system of arteries to the other.

The anastomoses between the pulmonary vein and the veins which we term the "pleuro-hilar" veins provide a ready decompressive mechanism in cases of raised pulmonary venous pressure. Ferguson, Kobilak, and Deitrick (1944) suggested that in cases of mitral stenosis the blood flow might be reversed from the pulmonary vein to the bronchial vein and thence to the systemic circulation. The bronchial vein to which they refer is presumably the vein described by us as the pleuro-hilar vein. Bland and Sweet (1949) described an operation for the relief of tight mitral stenosis based in principle upon these decompressive anastomoses. Hughes (1944) 
described two cases of anomalous pulmonary veins seen in mitral stenosis ; it is possible that he was describing dilated pleuro-hilar veins serving a decompressive role.

The true bronchial veins within the lung drain into the pulmonary circulation and anastomose freely with the pulmonary vein. Therefore pressure changes within the pulmonary vein will readily be transmitted to the true bronchial veins. Microscopically the bronchial venous radicles within the bronchial wall are arranged in two groups of very thin-walled vessels lying in the submucosa and on the outer aspect of the muscular layer. In the larger bronchi they are well separated from the bronchial lumen but in the bronchioles where the submucosa is very thin, these veins are situated very close to the lumen. In conditions associated with a raised pulmonary venous pressure congestion of these venous plexuses may partially occlude the bronchiole.

The hypertrophy and dilatation of the true bronchial vein which we found in emphysematous lungs associated with cor pulmonale may have been due to a sustained increase in pulmonary venous pressure. It is however possible that this hypertrophy followed a long-continued increase in blood flow through the bronchial vascular system.

No longer can the bronchial and pulmonary circulations be regarded as closed circuits. They communicate freely with each other on both the arterial and venous sides. The bronchial circulation is unique in that the artery arises from the systemic circulation, whilst its venous component drains into the pulmonary system.

\section{SUMMARY}

1. Anatomical studies of the vascular pattern encountered in 39 normal lungs and 24 diseased lungs are described.

2. True bronchial vessels are differentiated from vessels which supply and drain the sub-pleural and hilar structures.

3. Anastomoses between bronchial and pulmonary arteries in normal and diseased lungs have been described.

4. The anastomoses are most numerous in diseased lungs and are considered to be of functional importance.

5. The possible direction of blood flow through these anastomoses has been discussed. Their effect is considered to be of very great importance upon the haemodynamics of the pulmonary circulation in many diseased states.

6. The function of the pleuro-hilar veins in cases of pulmonary venous hypertension has been discussed and it is suggested that these veins act as an important decompressive mechanism.

We wish to express our gratitude to Professor Raymond A. Dart for granting us facilities in the Department of Anatomy, University of the Witwatersrand, where most of this work was done. Professor Joseph Gillman, Physiology Department, and Dr. T. Gillman, Anatomy Department, have rendered invaluable assistance in the choice of techniques, and in the preparation of this paper. Mr. L. Fatti, Thoracic Surgical Unit, Baragwanath Hospital, supplied the operation material for injection and has constantly helped us with this investigation. The photography is the work of Mr. A. Hughes, Anatomy Department, University of the Witwatersrand. 


\section{REFERENCES}

Bland, E. F., and Sweet, R. H. (1949). J. Amer. med. Ass., 140, 1259.

Bloomer, W. E., Harrison, W., Lindskog, G. E., and Liebow, A. A. (1949). Amer. J. Physiol., 157, 317 .

Braus, H. (1934). Anatomie des Menschen, 2nd ed., vol. 2, p. 203. Berlin.

Brenner, O. (1935). Arch. intern. Med., 56, 211.

East, T., and Barnard, W. G. (1938). Lancet, 1, 834.

Fatti, L., and Gilroy, J. C. (1949). Brit. Heart J., 11, 398.

Ferguson, F. C., Kobilak, R. E., and Deitrick, J. E. (1944). Amer. Heart J., $28,445$.

Hill, E. C. (1929). Bull. Johns Hopk. Hosp., 44, 248.

Hayek, H. von (1940). Anat. Anz., 89, 216. (1940). Z. Anat. EntwGesch., 110, 412.

Hughes, C. W., and Rumore, P. C. (1944). Arch. Path., 37, 364.

Keith, A. (1909). Lancet, 1, 745.

Liebow, A. A., Hales, M. R., and Lindskog, G. E. (1949). Amer. J. Path., $25,211$.

Mauer, G. (1941). Frankfurt. Z. Path., 55, 208.

Merkel, H. (1941). Virchows Arch., 308, 303.

Miller, W. S. (1947). The Lung, 2nd ed. Springfield.

Moore, R. A. (1929). J. tech. Meth., 12, 55.

Puckett, W. O., and Neumann, C. P. (1940). Anat. Rec., 78, 105.

Rivkind, A. V. (1949). Abstr. World Med., 5, 718. Abstr. No. 2486.

Verloop, M. C. (1948). Acta anat., Basel, 5, 171.

Virchow, R. (1851). Virchows Arch., 3, 427.

Wood, D. A., and Miller, M. (1938). J. thorac. surg., 7, 649.

Zuckerkandl, E. (1883). S.B. Akad. Wiss. Wien., 87, Part 3, p. 171.

Zwaag, G. L. van der (1940). Sclerosis arteriae pulmonalis primaria. Thesis, Groningen. 